\title{
PROTECTED THERMOELEMENTS
}

By Arthur W. Gray, Assistant Physicist

Although it is customary to mount industrial thermoelements in protecting tubes provided with convenient heads for attaching the leads to the apparatus employed for measuring the electromotive force, the wires of the average thermoelement to be seen in a physical or chemical laboratory are either entirely unprotected or else merely have portions adjacent to the junctions inserted in glass or porcelain tubes. Such an arrangement may be sufficient for temporary purposes, but it is by no means the best for everyday use. Kamerlingh Onnes ${ }^{1}$ has pointed out some of the advantages to be derived from properly protecting laboratory thermoelements, and has described in great detail the protected elements regularly used in the Leiden laboratory. ${ }^{2}$

Protected thermoelements have also been described by White ${ }^{3}$ and by the present writer. ${ }^{4}$ This paper deals with a type of mounting which has been gradually developed at the Bureau of Standards. It is applicable to elements for either high or low temperatures, and experience has shown it to be a great convenience in addition to giving adequate protection against damage by contamination or by mechanical strains.

The complete thermoelement with its ice bottle is shown in Fig. I just as it appears when ready for use; the three main parts are separated in Fig. 2; while a vertical section of the ice end is given by Fig. 3. The element represented is of copper and constantan. The slight modifications necessary for a platinum, platinum-rhodium element will be obvious.

1 H. Kamerlingh Onnes, Leiden Communication No. 27, p. $23 ; 1896$.

2 H. Kamerlingh Onnes, Leiden Communication No. 27, p. 20, 1896; H. Kamerlingh Onnes and C. A. Crommelin, Leiden Communication No. 89, r903; No. 953, r906.

${ }^{3}$ W. P. White, Journ. Am. Chem. Soc., 36, p. 2303; r9I4.

4 A. W. Gray, this Bulletin, 10, p. 464; r9r4. 
The silk insulation is left on the copper and the constantan wires except within the Jena combustion glass tube which covers the temperature-determining end. Within this tube the silk is removed from all but a few centimeters at the end farthest from the junction, and is replaced by a glass capillary surrounding one of the wires, because this thermoelement is used for temperatures up to over $300^{\circ} \mathrm{C}$. As a precaution the silk-covered portions of the wires are treated to a bath of hot paraffin to improve the insulation. The glass protecting tube is cemented with de Khotinsky cement into a sleeve soldered to one end of a soft copper tube through which the wires pass to the head at the ice-junction end. The sleeve $A$, soldered to the ice end of this copper tube, screws into a head, $B$, of mahogany which has been prepared by keeping in paraffin at about $120^{\circ} \mathrm{C}$ until bubbles of steam escaping from the wood cease to be visible. This head contains the binding posts $C$ and $D$ for receiving the leads to the potentiometer (or galvanometer) that is used for measuring the electromotive force by which the temperature is determined. Each of these binding posts is a single piece of round copper rod with an axial hole at its upper end to receive the lead wire and an extension at its lower end for attachment to one of the wires from the thermoelement. The posts are recessed in the head to avoid danger from parasitic thermal electromotive forces.

The insulating head, $B$, screws into a short brass tube, $E$, which is provided with a knurled collar, $F$, at the top and is closed at the bottom except for a central hole through which the glass tube, $G$, extends down into the middle of the ice bath. The lower end of this tube is drawn out thin and contains the thermoelement junction, $H$. The copper wires from the ends of the constantan wire are soft soldered to the extensions of the binding posts, $C$ and $D$, as indicated. In order to avoid temperature gradients across these soldered joints, very little solder is used, and, in addition, each wire, covered with its silk insulation right up to the joint, is tightly wrapped several times around the binding-post projection and then embedded in paraffin. Fused around the upper end of the tube, $G$, is a glass cup, $I$, which holds phosphorus pentoxide covered by glass wool and by the paraffin-soaked fiber washer $J$. The cup, $I$, is cemented by means of sealing wax into the brass tube $E$. 
Bull. Bur. Standards, Vol. 13
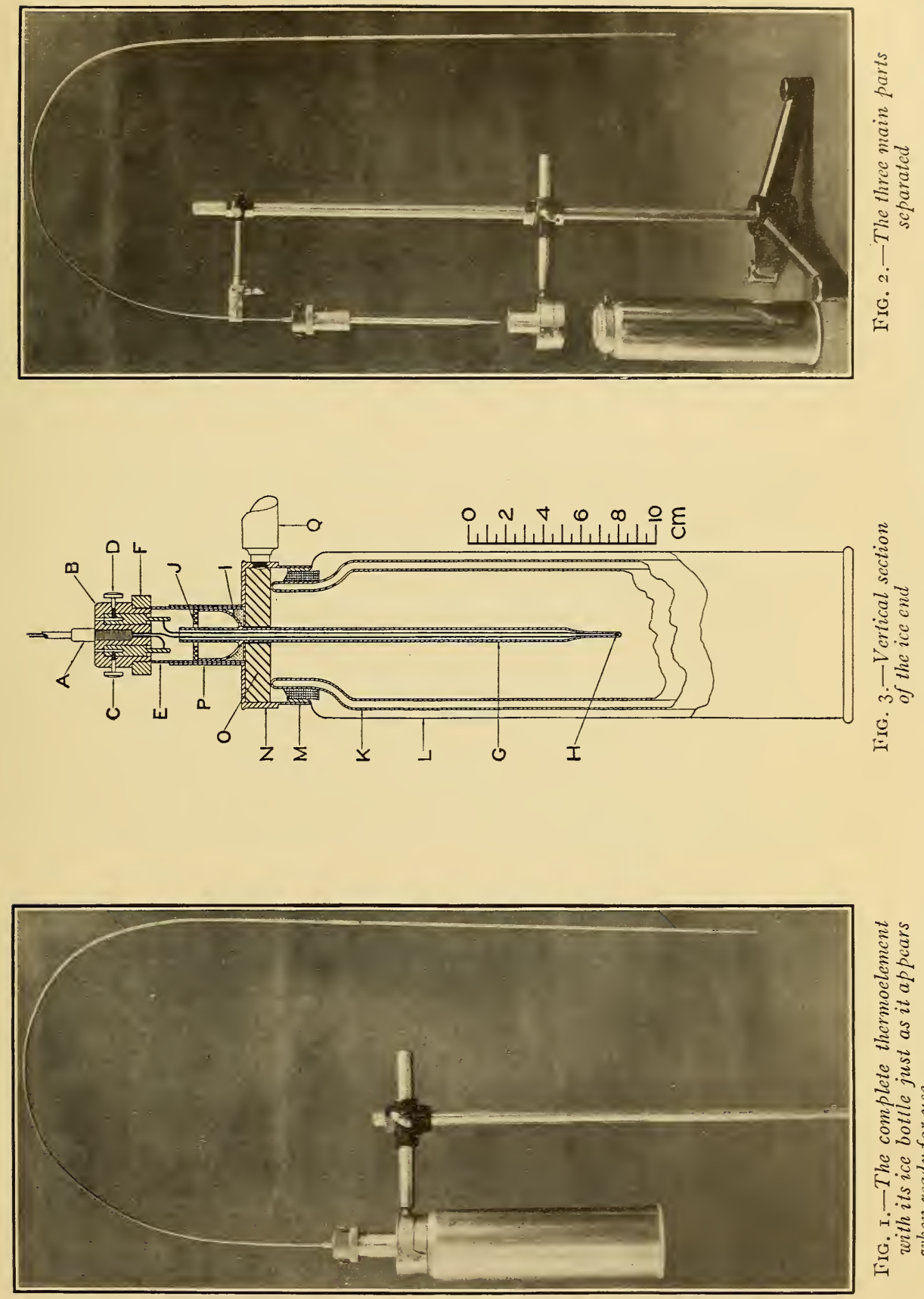

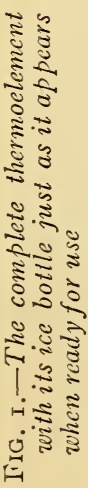


The ice surrounding the junction $H$ is contained in a I-quart "Caloris" vacuum jar, $K$, which comes protected by a thin nickelplated case, $L$. Around the top of this case is soldered a stout brass ring provided with two radial projecting pins (shown in Fig. 2 but not in Fig. 3) that form part of the bayonet joint from which the ice bottle is suspended from the cap $N$. A spring (visible in Figs. I and 2.) prevents the bayonet joint from accidentally coming loose.

Lining the cap, $N$, is the flat paraffin-soaked cork $O$, against which the vacuum jar, $K$, is pressed upward by a spiral spring in the bottom of the case $L$. Extending upward from the top of $N$ is a split tube $P$, into which the tube, $E$, of the thermoelement head fits with moderate friction. The whole assemblage is supported by the rod $Q$, and is made to fit the standard laboratory clamps.

The soft copper tube uniting the two ends of the thermoelement is flexible enough to permit bending, and yet is rigid enough to retain whatever shape is given to it. The wires within are thoroughly protected from damage by sharp bending, which is liable to introduce heterogeneity. Vacuum bottles of the type described frequently retain ice in good condition for two days. When it becomes necessary to renew the ice, a slight turn of the case, $L$, frees the bayonet joint and permits lowering of the ice container without disturbing anything else.

A multiple thermoelement divided into two equal parts as advocated by White ${ }^{5}$ can also be conveniently protected by the mounting just described if another pair of binding posts is provided.

WASHINGTON, January 27, I9I6. 